\title{
Shape Transformations of Giant Vesicles: Extreme Sensitivity to Bilayer Asymmetry.
}

\author{
K. BERNDL $\left({ }^{*}\right)$, J. KäS $\left({ }^{* *}\right)$, R. LIPOWSKY $\left({ }^{*}\right)\left({ }^{5}\right)$, E. SACKMANN $\left({ }^{* *}\right)$ \\ and U. SEIFERT $(*)\left({ }^{* 8}\right)$ \\ (*) Sektion Physik der Universität München \\ Theresienstr. 37, 8000 München 2, FRG \\ (**) Technische Universität München, Physik Department, Biophysics Group \\ 8046 Garching, FRG
}

(received 23 July 1990; accepted in final form 8 October 1990)

PACS. 82.70 - Disperse systems.

\begin{abstract}
Shape transformations of vesicles of lecithin (DMPC) in water are induced by changing the temperature which effectively changes the volume-to-area ratio. Three different routes are found which include i) symmetric-asymmetric re-entrant transitions from a dumbbell to a pear-shaped state, ii) the expulsion of a smaller vesicle (budding), and iii) discocytestomatocyte transitions. All of these shape transformations are explained within a model for the bending energy of the bilayer which assumes i) that the two monolayers do not exchange lipid molecules, and ii) that the two adjacent monolayers exhibit a small difference in their thermal expansivities which is easily produced, e.g., by residual impurities.
\end{abstract}

Artificial vesicles formed by lipid bilayers exhibit many different shapes. Apart from nearly spherical shapes, a variety of more exotic forms, such as, e.g., stomatocytes [1] or a necklace of small vesicles [2] has recently been observed. These shapes can be transformed into one another by changing the osmotic conditions, the composition of the lipid, or the temperature. Up to now, our understanding of these shape transformations has been rather limited. Indeed, all previous experiments have been performed with relatively complex systems containing, e.g., charged lipids, mixtures of different lipids, or additional solutes in the surrounding medium such as sugar. It was generally believed that these different ingredients play an essential role in determining the vesicle shape. Therefore, no attempt has been reported so far to relate these experimentally observed shapes in a systematic way to theoretical calculations.

In this paper, we study a very simple system consisting of one electrically neutral lipid in

(\$) Address after September 1st, 1990: Institut für Festkörperphysik, Forschungszentrum, Jülich, 5170 Jülich, FRG.

(ib) Address after October 1st, 1990: Department of Physics, Simon Fraser University, Burnaby, B.C. V5A 1S6, Canada. 
a Millipore water. Experimentally, we find that even for such a simple system a change in temperature can lead to a wide variety of shape transformations, see fig. 1-3. Theoretically, we find that these sequences of shape transformations can be explained by bending elasticity, provided one assumes a small asymmetry in the thermal expansivities of the two monolayers.

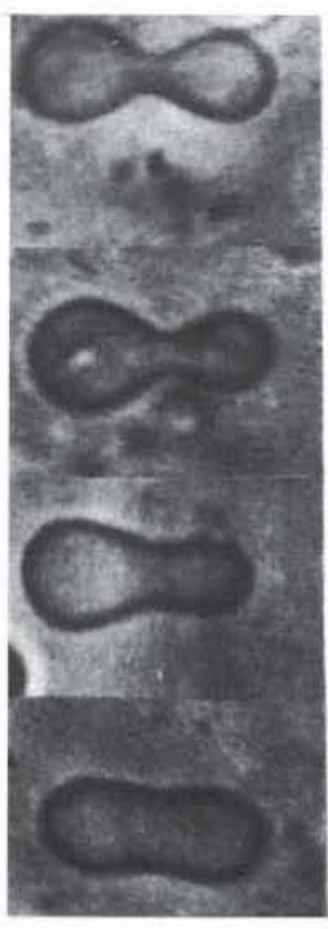

Fig. 1.

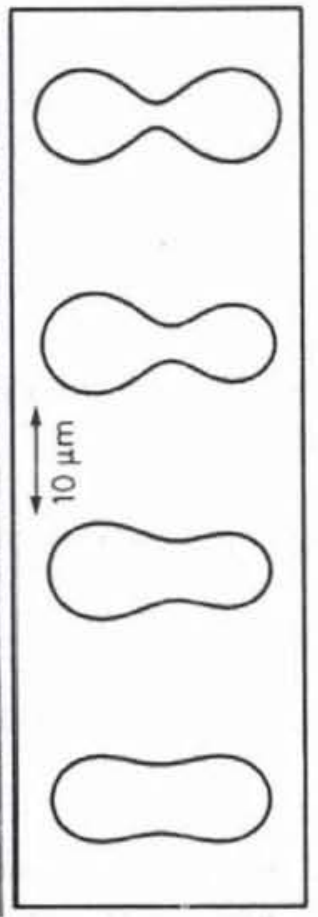

Fig. 2.

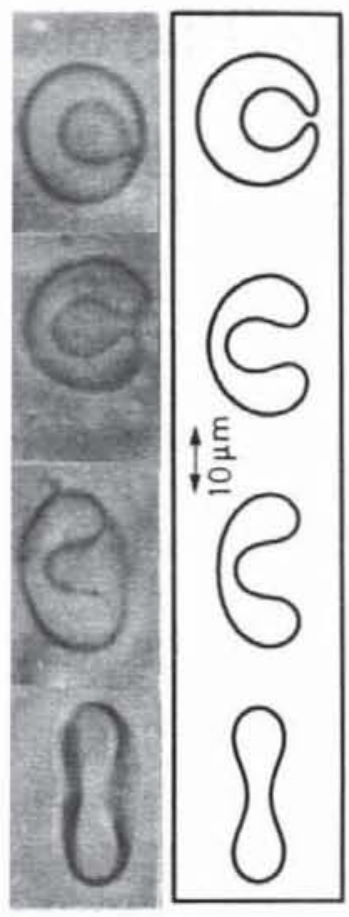

Fig. 3 .

Fig. 1. - Symmetric-asymmetric re-entrant transition: the shapes were measured at $T=30.7,32.6$, 40.0 and $44.3^{\circ} \mathrm{C}$. The calculated shapes correspond to a trajectory (4) with $v_{0}=0.78, \Delta a_{0}=1.1475$, $\gamma=0.00072$ and $b=640$.

Fig. 2. - Budding of a small vesicle: the shapes were measured at $T=31.4,35.5,35.6$ and $35.8^{\circ} \mathrm{C}$. The disclike object is due to an air bubble which migrates in the outer compartment of the measuring chamber. The calculated shapes correspond to a trajectory (4) with $v_{0}=0.9446, \Delta a_{0}=1.0305$, $\gamma=0.057$ and $b=1500$.

Fig. 3. - Discocyte-stomatocyte transition: the shapes were measured at $T=43.8,43.9,44.0$ and $44.1^{\circ} \mathrm{C}$. The calculated shapes correspond to a trajectory (4) with $v_{0}=0.65, \Delta a_{0}=1.0355, \gamma=-0.29$ and $b=1000$.

In fact, our theoretical considerations show that already a relative difference of the order of $10^{-3}$ in the thermal expansivities has a drastic effect on the shape of the vesicle. Such a minute difference is easily produced by residual impurities. On the other hand, one would have to prepare lipid bilayers for which the relative difference in the composition of both monolayers is of the order of $10^{-4}$ in order to obtain a unique sequence of shapes with increasing temperature. This seems hardly possible with the available experimental techniques.

In fig. 1-3, we present three different types of shape transformations which were i) found in our experiments, and ii) calculated with the theoretical model described below. The three theoretical sequences differ mainly in the value of the dimensionless parameter $\gamma$ which measures the asymmetry in the expansivity of the two monolayers. If the thermal expansivity of the outer monolayer is larger than the inner one with a relative difference 
$\gamma \geqslant 10^{-2}$, a small vesicle budds off the large vesicle. For $\gamma \approx 10^{-3}$, asymmetric pear-shaped states are attained, while a larger expansivity of the inner monolayer leads to the discocytestomatocyte transition and to the inclusion of a small cavity.

Our experiments were performed with vesicles of dipalmitoyl phosphatidyl choline (DMPC) of diameters larger than $10 \mu \mathrm{m}$. These were prepared in a separate test-tube. The lipid with a purity $>99 \%$ was dissolved in a solvent of $2: 1$ chloroform/methanol to produce a $1 \mathrm{mM}$ solution. Then, $60 \mu \mathrm{l}$ of this solution were distributed as a thin film on the inner surface of the test-tube in a vacuum chamber for one day. It is generally believed that this time is sufficient to obtain bilayers which are essentially free of solvent. Then the vesicles were swollen by filling the test-tube with $5 \mathrm{ml}$ distilled water prepared with a Milli-QSystem. After swelling, the vesicles were transferred into a special chamber which allows, for the first time, the observation of free vesicles with essentially no thermal convection. For that purpose, the chamber consists of an inner compartment which contains the vesicle and an outer compartment used for temperature control. The details of this chamber will be described elsewhere [3]. During the slow increase of the temperature $\simeq 0.2 \mathrm{~K} / \mathrm{min}$, the volume and the area of the vesicles were measured by a digital image processing system.

Our calculations are based on the bilayer coupling model of Svetina and Zeks [4] which represent a Legendre transformation of the spontaneous curvature model of Helfrich [5] $\left.{ }^{(}\right)$. The two monolayers are taken to have constant separation $d$. The shape of the vesicle is determined by the minimum of the bending energy, $F_{x}$,

$$
F_{\mathrm{x}}=(\kappa / 2) \oint\left(C_{1}+C_{2}\right)^{2} \mathrm{~d} A^{\mathrm{un}},
$$

which is expressed as an integral over the inner monolayer only since both monolayers are coupled $\left({ }^{2}\right)$. Here, $\boldsymbol{K}$ denotes the effective bending rigidity of the bilayer with $K \simeq 1.15 \cdot 10^{-19} \mathrm{~J}$ for DMPC [6]. The variables $C_{1}$ and $C_{2}$ are the two principal curvatures. Since we only consider shape transformations which do not change the topology of the vesicle, we can omit $a$ Gaussian curvature term. The area difference $\Delta A \equiv A^{\mathrm{ex}}-A^{\text {in }}$ of both monolayers is related to the total mean curvature of the inner monolayer via $\Delta A \approx d \oint\left(C_{1}+C_{2}\right) \mathrm{d} A^{\text {in }}$ for small $d$. The minimization of $F_{\mathrm{K}}$ is now performed for fixed area $A^{\text {in }}$, fixed enclosed volume $V$, and fixed area difference $\Delta A$. Thus, we assume that the exchange of lipid molecules between both monolayers can be ignored on experimentally relevant timescales.

For the model just described, we have theoretically determined the «phase diagram" [7], i.e. we have determined the axi-symmetric shapes of the lowest bending energy for experimentally accessible values of the dimensionless parameters $v$ and $\Delta a$, defined by

$$
v \equiv V /\left[(4 \pi / 3)\left(A^{\mathrm{in} / 4 \pi}\right)^{3 / 2}\right] \quad \text { and } \quad \Delta a \equiv \Delta A /\left[8 \pi d\left(A^{\mathrm{in} / 4 \pi}\right)^{1 / 2}\right] .
$$

For a sphere, one has $v=\Delta a=1$. This phase diagram is displayed in fig. 4. Some part of it has been previously described by Svetina and Zeks [4]. As two important novel features, we find i) an instability of the dumbbell shapes with respect to the up/down symmetry which leads to the pear-shaped states, and ii) new limiting shapes which look like two prolate ellipsoids sitting on top of each other.

(1) Even though both models are related via a Legendre transformation, the corresponding phase diagrams are rather different because the relevant energy is different; in particular, the shape transformations can change from first to second order, see ref. [7].

(2) It is convenient here to express all quantities in terms of the surface areas, $A^{\text {in }}$ and $A^{\text {ex }}$, rather than in terms of the neutral surface. 
In order to compare theoretical and experimental shapes, we introduce temperature trajectories into the phase diagram and determine the path $\Delta a=\Delta a(v)$ which corresponds to a change in temperature $T$. We now assume that the interiour and exteriour monolayer have different relative expansivities, $\alpha^{\text {in }}$ and $\alpha^{\text {ex }}$, as given by

$$
\alpha^{\text {in }} \equiv \frac{1}{A^{\text {in }}} \frac{\mathrm{d} A^{\text {in }}}{\mathrm{d} T} \quad \text { and } \quad \alpha^{\mathrm{ex}} \equiv \frac{1}{A^{\mathrm{ex}}} \frac{\mathrm{d} A^{\mathrm{ex}}}{\mathrm{d} T}, \quad \text { with } \alpha^{\mathrm{ex}}=(1+\gamma) \alpha^{\text {in }} .
$$

For simplicity, we neglect the small thermal expansivity of the enclosed water and assume that the volume of the bilayer given by $A^{\text {in }} d$ is temperature independent. Then the temperature trajectory follows from eqs. (2) and (3) as

$$
\Delta a(v)=\left(\frac{v_{0}}{v}\right)\left[\Delta a_{0}+b\left(\left(\frac{v_{0}}{v}\right)^{2 \gamma / 3}-1\right)\right]
$$

where $v_{0} \equiv v\left(T_{0}\right)$ and $\Delta a_{0} \equiv \Delta a\left(T_{0}\right)$ parametrize the initial shape at temperature $T=T_{0}$ and

$$
b \equiv A^{\mathrm{ex}}\left(T_{0}\right) /\left\{8 \pi d\left(T_{0}\right)\left[A^{\mathrm{in}}\left(T_{0}\right) / 4 \pi\right]^{1 / 2}\right\} .
$$

For $\gamma=0$, i.e. if the asymmetry were not present, the second term in (4) vanishes and a temperature trajectory in the phase diagram would be a simple hyperbola as given by $\Delta a(v)=\left(v_{0} / v\right) \Delta a_{0}$. This path is shown in fig. 4: starting, e.g., with a symmetric prolate ellipsoid, it immediately crosses the phase boundary $C^{\text {pear }}$ and enters the pear-shaped region; it then crosses again the line of continuous transitions $C^{\text {pear }}$ and finally meets the new type of limiting shapes at $L^{\text {dumb }}$. Note that such a path never enters the stomatocyte region.

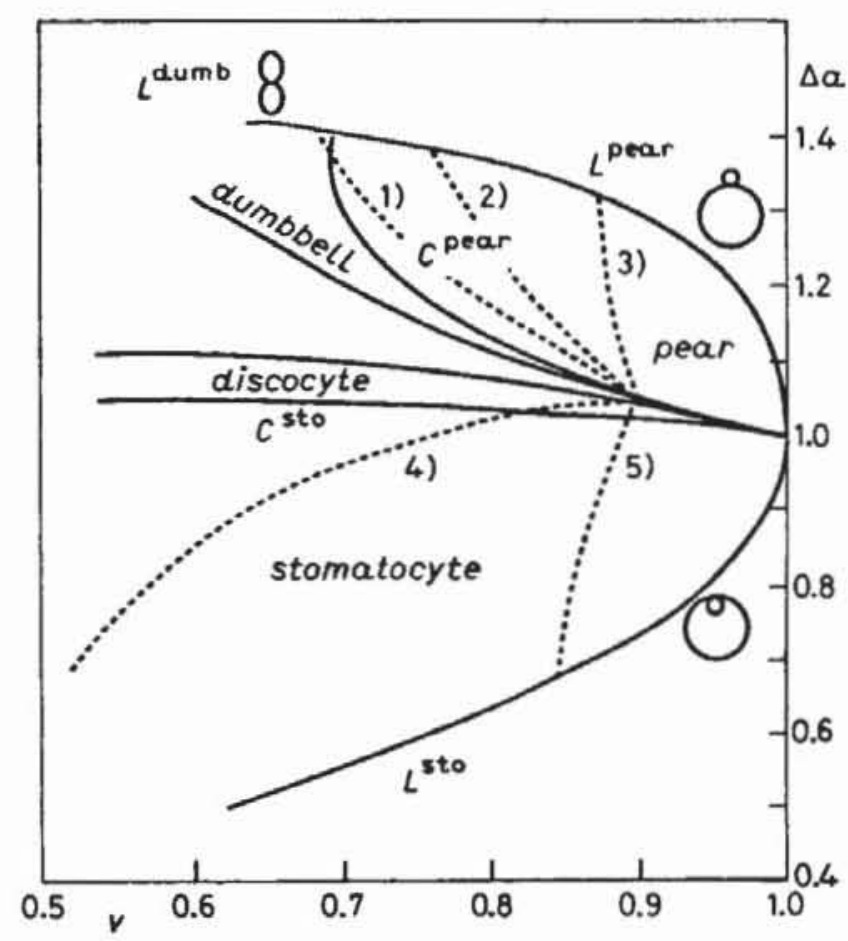

Fig. 4. - Phase diagram and temperature trajectories for the bilayer coupling model. This phase diagram shows the state of lowest energy for given $\Delta a$ and $v . C^{\text {pear }}$ and $C^{\text {ato }}$ denote lines of continuous transitions at which the up-down symmetry of the vesicle shape is broken. $L^{\text {pear }}, L^{\text {sto }}$ and $L^{\text {dumb }}$ denote limit shapes. The dashed lines 1)-5) represent temperature trajectories for asymmetry parameter $\gamma=0,0.001,0.01,-0.002$ and -0.01 , respectively, for fixed $b=900, v=0.9$ and $\Delta a_{0}=1.05$. 
For $\gamma \neq 0$, the second term in (4) contributes and one can reach many different shapes by changing the temperature, see fig. 4. As shown in this figure, a very small value of $\gamma$ has a very strong effect. This can be understood from an estimate of the parameter $b$ as given by (5): for the typical values $A^{\mathrm{ex}}\left(T_{0}\right) \simeq A^{\mathrm{in}}\left(T_{0}\right) \simeq 100 \mu \mathrm{m}^{2}$ and $d \simeq 5 \mathrm{~nm}$, one obtains $b \simeq 900$ ! Thus, an asymmetry of the order of $10^{-3}$ will already have a significant effect as shown in fig. 4. In this figure, paths for different values of $\gamma$ are displayed for the same initial state with $v_{0}=0.9$ and $\Delta a_{0}=1.05$ which corresponds to a prolate ellipsoid.

The asymmetry $\gamma$ cannot be measured or controlled directly in our experiment. A crude estimate, however, can be obtained by comparing the experimental with theoretical shapes. The calculated shapes shown in fig. 1-3 lie on a trajectory (4) within the experimentally observed temperature intervals. For fig. 1 and fig. 3 , the parameters $b, v_{0}, \Delta a_{0}$ and $\gamma$ were obtained as follows: the measured area $A$ and volume $V$ of the first shape at temperature $T_{0}$ determines $v_{0}$ and $b$, where we used $A^{\mathrm{m}} \simeq A^{\mathrm{ex}} \simeq A$ and $d \simeq 5 \mathrm{~nm}$. The area difference $\Delta a_{0}$ is chosen to fit the experimental shape. The measured temperature interval $T_{\mathrm{f}}-T_{0}$ between the final and the initial shape determines $v\left(T_{f}\right)$ via $v(T)=v_{0} \exp \left[-(3 / 2) \alpha^{\text {in }}\left(T-T_{0}\right)\right]$, with [8] $\alpha^{\text {in }}=6 \cdot 10^{-3}$. Once again, $\Delta a\left(T_{f}\right)$ is fitted to the experimental shape, which finally determines the value of the asymmetry parameter $\gamma$. For fig. 2, we first determined $v\left(T_{\ell}\right)$ from the last shape and then $v\left(T_{0}\right)$ from the measured temperature difference to the second shape.

The relatively large value of $\gamma \simeq-0.29$ thus obtained for the sequence shown in fig. 3 , results from the small temperature interval in which the shape transformation happens. The physical origin of this relatively large value of $\gamma$ is not clear.

In fig. 2, we have shown budding of one smaller vesicle. Since the small vesicle remains connected with the larger one, the topology has not changed. Therefore the Gaussian curvature term does not contribute to this type of budding. With a further increase in temperature, additional small vesicles appear forming a necklace. Theoretically, these shapes can be obtained by continuing the trajectory beyond $L^{\text {pear }}$.

The shape equations for the bilayer coupling model are equivalent to those of the spontaneous curvature model. Therefore, the same shapes are obtained in both models and one might expect that the observed shape transformations can also be explained in the spontaneous curvature model. We have also determined the phase diagram in this ensemble, as will be discussed in detail elsewhere [7]. For zero spontaneous curvature, $C_{0}=0$, which would be the most natural choice, we find a first-order transition between a dumbbell and a discocyte shape at $v \simeq 0.65$ and a further discontinuous transition to a stomatocyte shape at $v \simeq 0.59$. The latter value for $v$ is significantly lower than the $v$-value observed in our experiments. Furthermore, in order to obtain a budding trajectory, one would have to postulate a spontaneous curvature $C_{0}>2 \sqrt{2} /(A / 4 \pi)^{1 / 2}$. Even for such a value of $C_{0}$, the budding trajectory would exhibit a pronounced first-order transition between the dumbbell and a pear-shaped state with a very narrow neck in contrast to what has been experimentally observed. Likewise, shapes along a re-entrant trajectory as in fig. 1 have a significantly narrower neck than those observed in our experiment. Finally, the transition from the symmetric to the pear-shaped state is discontinuous in the $C_{0}$-model contrary to our observation.

Based on such a comparison, we conclude that the bilayer coupling model together with our hypothesis of a small asymmetry in the thermal expansivities is more appropriate in order to describe the different routes found in our experiment.

This work was supported by the Deutsche Forschungsgemeinschaft through the Sonderforschungsbereich No. 266. 


\section{REFERENCES}

[1] Sackmann E., Duwe H. P. and Engelhardt H., Faraday Discuss. Chem. Soc., 81 (1986) 281.

[2] Evans E. and Rawicz W., Phys. Rev. Lett., 64 (1990) 2094.

[3] KäS J. and SACKMANN E., to be published.

[4] Svetina S. and Zeks B., Eur. Biophys. J., 17 (1989) 101.

[5] Helfrich W., Z. Naturforsch C, 28 (1973) 693; Deuling H. J. and Helfrich W., J. Phys. (Paris), 37 (1976) 1335.

[6] Duwe H. P., Käs J. and Sackmann E., J. Phys. (Paris), 51 (1990) 945.

[7] SEIFERT U., BERNDL K. and LIPOWSKY R., to be published.

[8] Evans E. and Needham D., J. Phys. Chem., 91 (1987) 4219. 